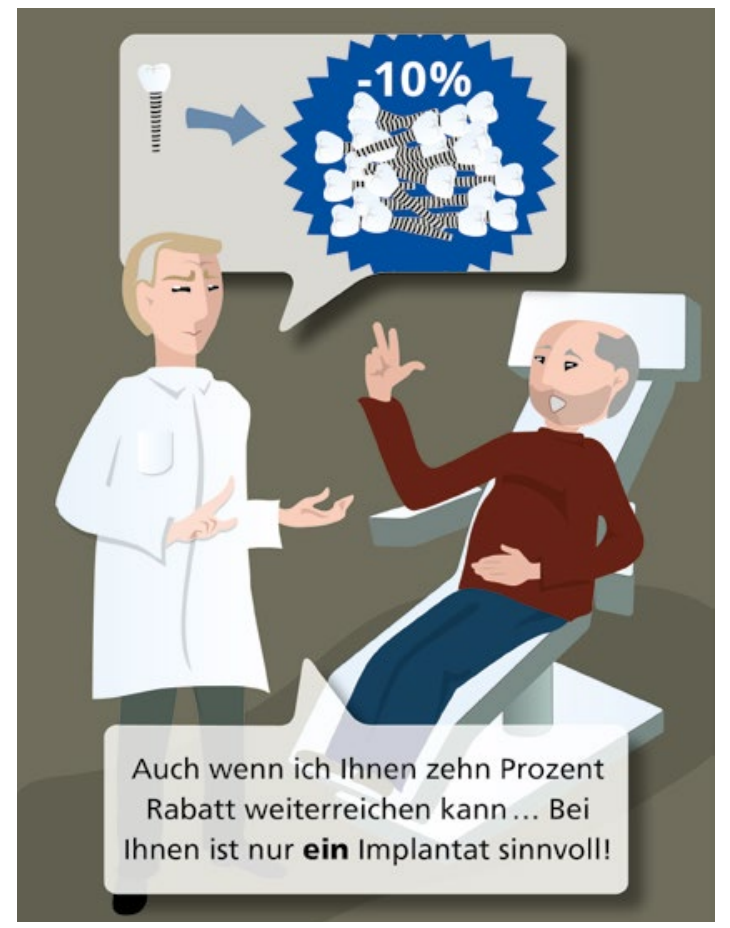

\section{Rabatte für Medizinprodukte}

Zahnarzt Dr. Hohlzahn hat bei einem Implantathersteller einen Rabatt ausgehandelt - und nicht nur das: Wenn er 500 Implantate abnimmt, bekommt er zehn Prozent Rabatt. Das ist ein guter Deal. Allerdings nur dann, wenn Rabatte grundsätzlich gewährt werden. Wenn nur Dr. Holzahn in den Genuss kommt, und er sich dadurch noch bemüßigt sieht, mehr Implantate zu verkaufen, dann bekommt die Sache den Geschmack der Vorteilsnahme. Gibt der Zahnarzt noch dazu den Rabatt nicht an den Patienten weiter, geht es vollends auf Korruptionskurs. Grundsätzlich darf nie der Eindruck entstehen, dass sachfremde Entscheidungen Einfluss auf die Behandlung eines Patienten nehmen.

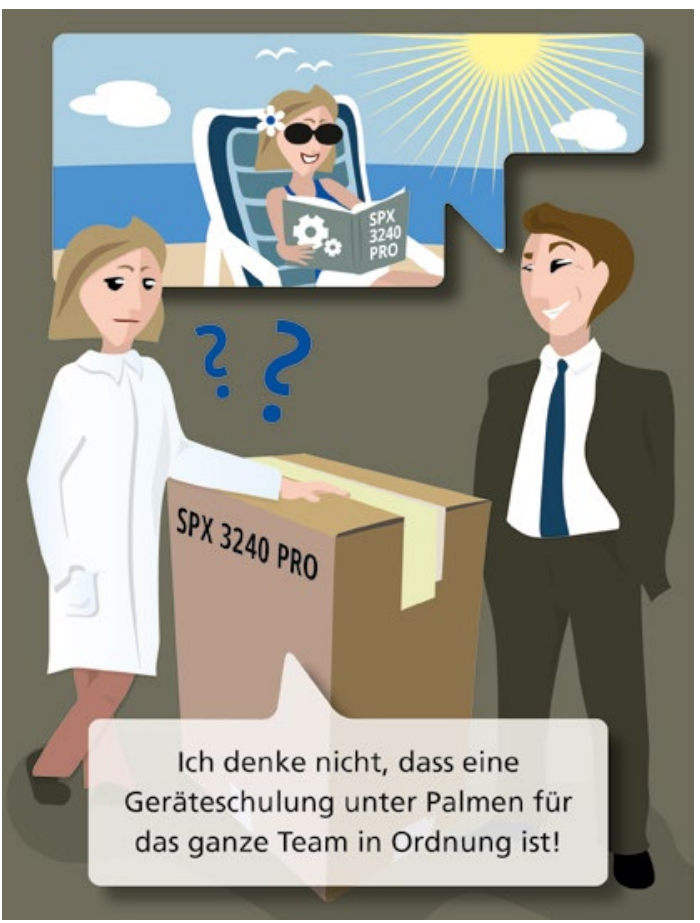

politik rausragenden Bedeutung des Grundrechts der Berufsfreiheit nicht akzeptabel, dass die Ärzte aus Angst vor Strafverfolgung zu einer vorsichtigen Grundrechtsausübung ,in dubio contra libertate" gezwungen werden. Hierunter dürfte auch die Innovationskraft der Branche in Bezug auf die Entwicklung neuer Praxis- und Kooperationsmodelle leiden.

DFZ: Was sehen Sie noch kritisch an dem aktuellen Entwurf? Pragal: Der Tatbestand umfasst jetzt drei Alternativen, wobei $\$ 299$ a Abs. 1 Nr. 1 StGB meines Erachtens alle relevanten Erscheinungsformen abdeckt. Der Gesetzgeber sollte daher zunächst auf den unnötigen „Auffangtatbestand“ des $\$ 299$ Abs. 1 Nr. 2 StGB-E verzichten. Denn die angeblichen Lücken des Abs. 1 Nr. 1 bei Monopolen und nichtindizierten Behandlungen sind nicht praxisrelevant.

\section{DFZ: Was kritisieren Sie an § 299a Abs. 2 StGB-E?}

Pragal: Diese Variante ist eigentlich geschaffen worden, um die eigene unternehmerische Sphäre des Arztes von vornherein vom Tatbestand abzugrenzen, was zu begrüßen ist, beispielsweise den Kauf eines Behandlungsstuhls. Er setzt daher voraus, dass Korruption beim Bezug von Arznei-, Heil- oder Hilfsmitteln oder Medizinprodukten nur stattfinden kann, wenn diese Gegenstände „zur Abgabe an den Patienten bestimmt sind“.

DFZ: Wie beurteilen Sie die Änderungen im Sozialgesetzbuch V in Bezug auf die Berichtspflichten und den erweiterten Erfahrungsaustausch unter Beteiligung der Staatsanwaltschaft?

Pragal: Ich sehe die Beteiligung der Staatsanwaltschaft positiv,

\section{Fortbildung unter Palmen}

Zahnärztin Dr. Sonnenschein hat ein neues Gerät für die Praxis gekauft. Ganz modern, sehr nützlich. Der Hersteller lädt die Zahnärztin und ihr gesamtes Team zum Seminar mit Erklärung des Gerätes zu einem viertägigen Aufenthalt in ein 5-Sterne-Hotel auf Mallorca ein. Schöne Sache, denkt sich Dr. Sonnenschein. Ist aber nicht so, denn in diesem Fall ist ganz klar die Verhältnismäßigkeit nicht gewahrt. Der Verkäufer gewährt Vorteile, die einer normalen Geschäftsbeziehung nicht entsprechen. Eine Einarbeitung des Personals kann der Hersteller in der Praxis anbieten, und diese wird vermutlich einen Tag nicht überschreiten. Eine Fortbildung unter Palmen dürfte in den seltensten Fällen gerechtfertigt sein.

da dies für einen Wissenstransfer sorgen wird, der auch Missverständnisse vermeiden wird, zum Beispiel hinsichtlich der berufsrechtlichen Zulässigkeit von Kooperationen oder Ähnlichem. Hier geht es nicht darum, dass die Staatsanwaltschaft im Einzelfall „auf die Spur gesetzt“ wird. Die Berichte können angesichts der zu erwartenden geringen Fallzahlen sogar zur amtlich bestätigten Imagewerbung für die Ärzteschaft werden.

DFZ: Worauf sollte der FVDZ im weiteren Verlauf des Gesetzgebungsverfahrens noch Wert legen?

Pragal: Der FVDZ sollte insbesondere darauf achten, dass der Gesetzgeber bei seiner richtigen Entscheidung bleibt, weder die Telefonüberwachung zuzulassen noch den neuen Korruptionstatbestand in den Katalog der Geldwäschetaten einzubeziehen. Letzteres kann nämlich im Extremfall zur „Kontamination“ ganzer Praxen wegen einzelner oder einiger weniger Verfehlungen führen.

DFZ: Wo sehen Sie denn zukünftig konkret Strafbarkeitsrisiken? Was muss der einzelne Zahnarzt Ihrer Meinung nach jetzt tun?

Pragal: Hochkritisch ist der Erhalt jeglicher Vorteile von externen Laboren. Dies betrifft nicht nur Rückvergütungen, sondern auch zum Beispiel Vergütungen für Vorträge oder Beratung durch den Zahnarzt oder Beteiligungen an Laboren. Auch Kooperationen zwischen Zahnärzten und Ärzten anderer Fachrichtungen sollten von einem qualifizierten Rechtsanwalt auf eine unzulässige Zuweisung gegen Entgelt hin untersucht werden, die künftig strafbar sein können. Da das Gesetz vermutlich Anfang 2016 in Kraft treten wird, bleibt für eine Anpassung nicht mehr viel Zeit.

Interview: DFZ-Redaktion 